\title{
Relocation of specific endothelial features with the clinical specular microscope
}

\author{
EMIL S. SHERRARD AND ROGER J. BUCKLEY \\ From Pocklington Eye Transplantation Research Unit, Institute of Ophthalmology, \\ and Moorfields Eye Hospital, City Road, London
}

SUMMARY The selection and later relocation of specific areas of the in-vivo human corneal endothelium at relatively high magnification with a clinical specular microscope are demonstrated by case examples. Relocation of an area of the endothelium is greatly facilitated by the large field of view, the reduction of eye movement, and the presence of posterior corneal rings, induced by applanation of the cornea, which serve as a target system.

Clinical specular microscopy of the corneal endothelium has often been criticised as a nonselective method because the areas of the endothelium which may be observed and photographed are arbitrary and difficult to relocate. The very small area of the endothelium included in any one field of view with most contact specular microscopes, and the low initial magnification of the noncontact instruments, combined with the continuous rapid fine movements of the living eye, virtually disallow the selection of specific regions of the endothelium for study. Moreover the interrelationships of various fields of view and of any particular endothelial features therein are essentially unknown. Rosenblum and associates ${ }^{1}$ have recently stressed the importance of this sampling limitation of clinical specular microscopical technique.

We have reported elsewhere ${ }^{2-4}$ that the Pocklington (Keeler-Konan) contact clinical specular microscope provides a considerably larger field of view $\left(1.18 \times 0.79 \mathrm{~mm}^{2}\right)$ of the corneal endothelium than most other available instruments, at an adequate magnification $(130 \times)$ through the camera viewfinder, and that by virtue of its being used in conjunction with a high water content contact lens (Duragel 75 or Optima 81), and the other special features, it reduces considerably the movements of the living eye.

We have described ${ }^{25}$ the formation of a concentric series of wrinkles in the posterior corneal layers which result as an artefact of applanation with the microscope's objective cone and noted that they occur in

Correspondence to Dr E. S. Sherrard, Institute of Ophthalmology, Judd Street, London WC1 9QS fixed positions surrounding the central area of the cornea, not the area of applanation. We have termed these the posterior corneal rings (PCRs) and identified them numerically: the innermost ring is PCR1 and typically has an approximate diameter of $3 \mathrm{~mm}$ horizontally and $2.5 \mathrm{~mm}$ vertically in the normal eye. Later observations have indicated that PCRs proper occur in the adult cornea while a simplified formPCR precursor-appears only in younger individuals. The brightly reflecting ridges of the PCRs or the dark shadows of their precursors can be seen clearly, even in the presence of a substantial degree of stromal haze, when the endothelial mosaic is far out of focus. Hence they provide an obvious indication of the plane of the endothelium upon which to focus and, by virtue of their fixed positions, an accurate target for the location and relocation of precise areas of the endothelium. We earlier contended that, given this target system, the larger field of view and the reduced movements of the eye, observations of precise, selected areas of the human corneal endothelium in vivo are now a reality. ${ }^{2}$ Subsequently we have been able to support this contention and here present some illustrated examples.

\section{Case reports}

CASE 1

The purportedly normal corneal endothelium of a volunteer subject was employed as test material in the evaluation of a small field contact clinical specular microscope in July 1979. During uncontrolled 


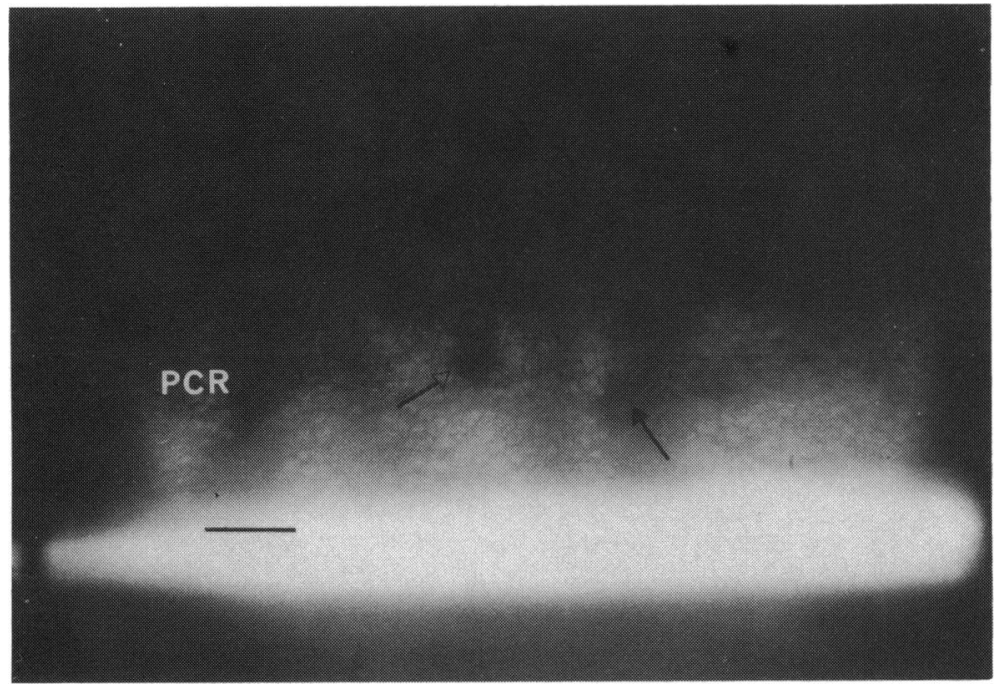

Fig. 1 Case 1. Specular photomicrograph of in-vivo corneal endothelium showing 2 small guttata (arrows) and PCR segment. With small field specular microscope. Bar $=0.1 \mathrm{~mm}$.

scanning two small dark 'lesions' lying close together were observed and photographed (Fig. 1). The location of the lesions was known to be only in the temporal side of the right cornea. Slit-lamp examination revealed a normal endothelium apart from 2 guttata in this same area. Eighteen months later the original specular photomicrograph was scrutinised, and it was apparent, from knowledge of posterior corneal rings gained in the meantime, that the guttata were situated at approximately the 9 o'clock position close to an unknown PCR (Fig. 1). The cornea was re-examined but now with the large field specular microscope (the Pocklington) and by tracing round the PCR system to the 9 o'clock position the guttata were relocated and photographed (Fig. 2) in a timed search period of 26 seconds.
One of the guttata (solid arrow) is virtually lost in a posterior corneal wrinkle in both Figs. 1 and 2 and defies comment. The other (open arrow) appears larger in Fig. 1 than in Fig. 2. This is probably an illusion due to poor resolution and focusing in Fig. 1, for a comparison of the endothelial mosaic around and between the guttata on a cell-for-cell basis in the 2 photomicrographs shows only minimal change.

Controlled scanning of the endothelium at the second specular microscopical examination revealed no other abnormalities.

\section{CASE 2}

An 18-year-old girl was referred to the clinic for specular microscopical evaluation of the endothelium of both corneas. A slit-lamp examination had

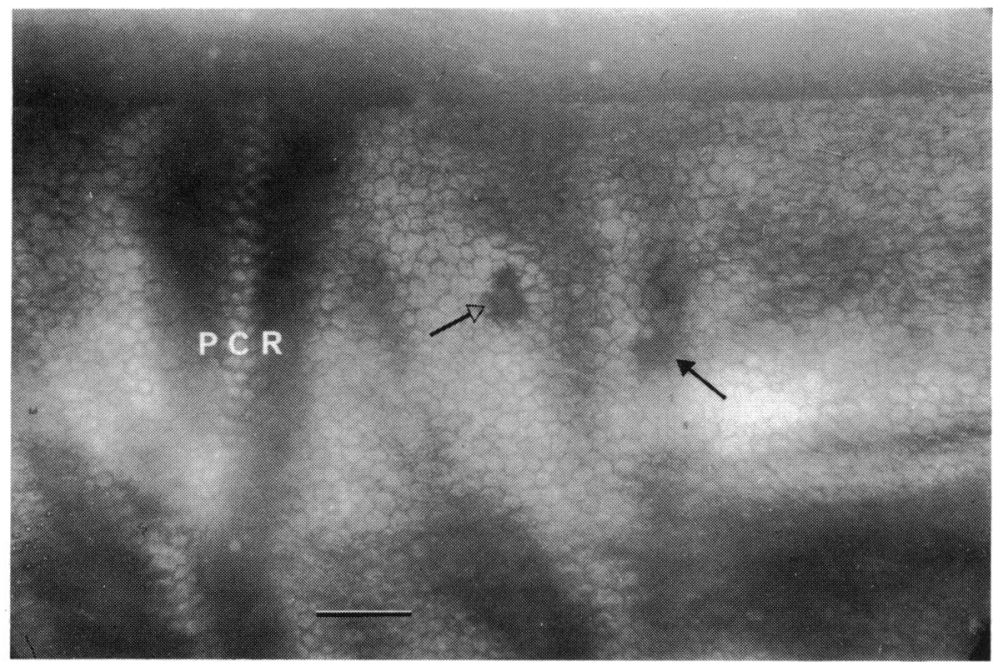

Fig. 2 As in Fig. 1 but with large field specular microscope and 18 months later. The configuration of the PCR identifies the same area of the endothelium in Figs. 1 and 2. The unlabelled linear shadows are random posterior corneal wrinkles. 
Fig. 3 Case 2. Specular photomicrograph from left eye showing medium and small sized lesions in the corneal endothelium. The former contain cells, the latter are intra-(arrow) and intercellular. On day 0. Bar $=0.1 \mathrm{~mm}$. Squares: see caption to Fig. 4.

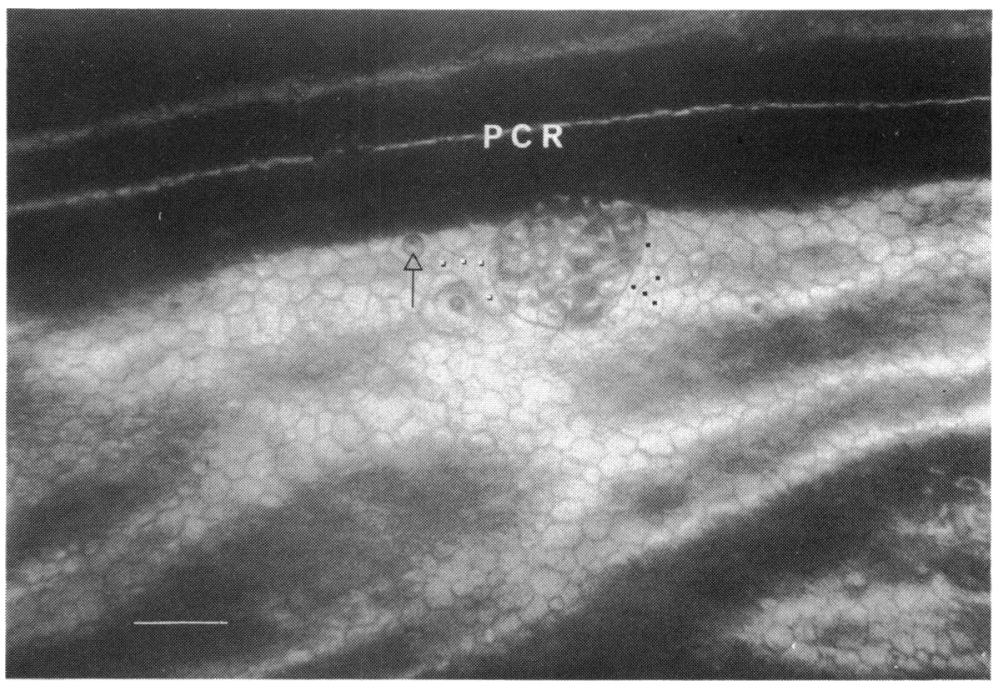

revealed numerous clearly defined endothelial lesions; most of these were essentially circular and exhibited a considerable range in size. Large field specular microscopy expanded these observations by demonstrating that the cells in general were irregularly enlarged and that the larger lesions contained endothelial cells within them while the smallest appeared as both intra- and intercellular inclusions (Fig. 3). The nature of the endothelial changes could not be determined, but after consideration of the other general and ocular signs and by comparison with other examples an infective aetiology was suggested. Samples of each morphologically distinct lesion were selected in both eyes according to their location in relation to the PCRs, and photographed. Figs. 3, 5, and 7 show some of them. Then at weekly intervals the same lesions were relocated, by reference to the PCRs, studied and rephotographed. Figs. 4, 6, and 8 show the same areas of endothelium and the same lesions as in Figs. 3, 5, and 7 respectively, after 21 days. During this time the other ocular signs subsided, but the specular photomicrographs show no resolution of the endothelial abnormality. Indeed, cell-by-cell comparison reveals enlargement of the lesion in one instance (see figure captions). It is certain that the lesions photographed at 0 and 21 days are the same and not other similar entities which had arisen meanwhile or were overlooked originally, because of their positions relative to the PCRs. Observations of this case are continuing.
Fig. 4 As in Fig. 3 but on day 21. The association of the lesions with the PCR (i.e., 11.30 o'clock on $P C R 2)$ shows that the field is the same in both photographs (Figs. 3 and 4). The largest lesion is seen to have extended inferotemporally during the 3-week interval between photographs (Figs. 3 and 4), with loss of cells marked with black squares in Fig. 3, and alteration of shape of cells marked with white squares in Fig. 3. Bar $=0.1 \mathrm{~mm}$.

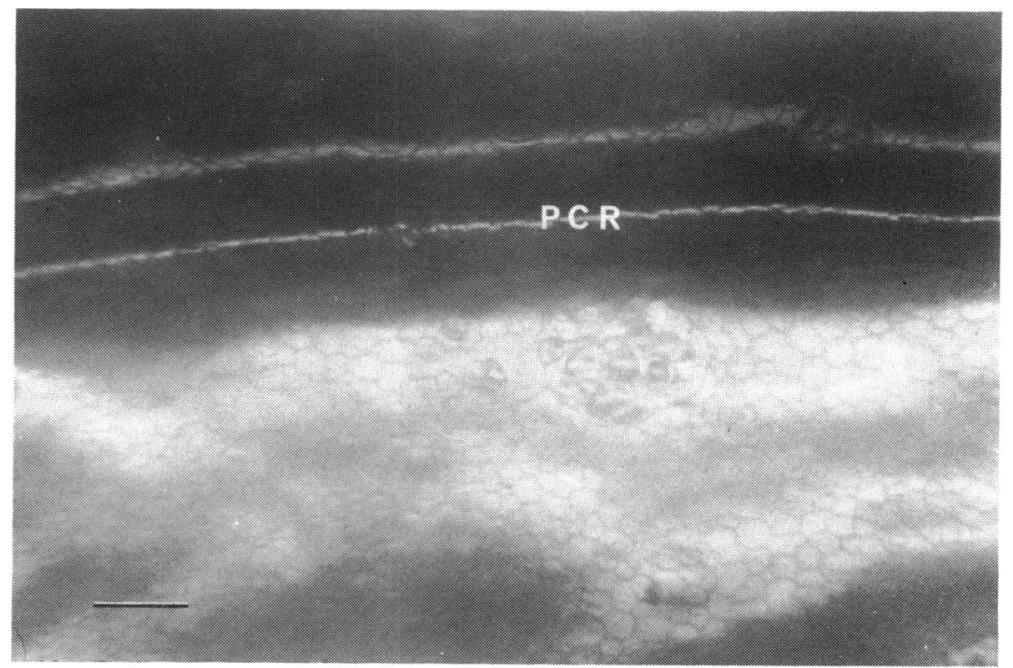




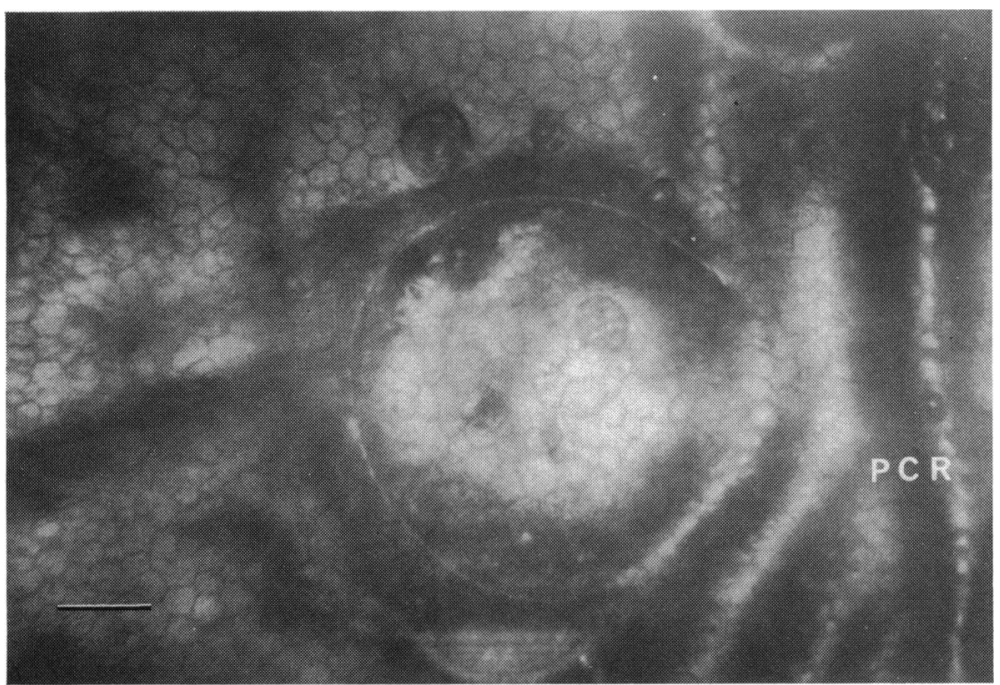

Fig. 5 Case 2. Specular photomicrograph of corneal endothelium from left eve showing large circular lesions containing cells. On day 0. Bar $=0.1 \mathrm{~mm}$.
CASE 3

The endothelium of the left eye of a 57-year-old woman with heterochromic cyclitis was examined with the large-field specular microscope. Many and various abnormal features were observed and photographed. Some appeared as very large dark areas with the surrounding endothelial cells elongated towards them. One of these (Fig. 9) was selected for follow-up study, because of its potential ease of relocation and identification among other similar lesions, by its position relative to the PCRs. Fig. 10 shows the selected lesion, the same as in Fig. 9, purposely offcentred to record that it is at the 5 o'clock position on PCR1. Seven and a half months later the same lesion was rapidly found and rephotographed. Fig. 11 shows its appearance at this time. Comparison of Figs. 9 and 11 reveals that the lesion has enlarged slightly and that some of the surrounding cells have lost or gained small dark inclusions, and that all the cells have changed shape so as to be unrecognisable from one picture to the other.

\section{CASE 4}

On 13 March 1980 a test photomicrograph (Fig. 12) was taken of the normal cornea of a volunteer subject and by comparison with the PCR segment included in it the same area was relocated and photographed on 8 January 1981 (Fig. 13).

The configuration of the PCR and associated posterior corneal wrinkles is strikingly similar in the 2

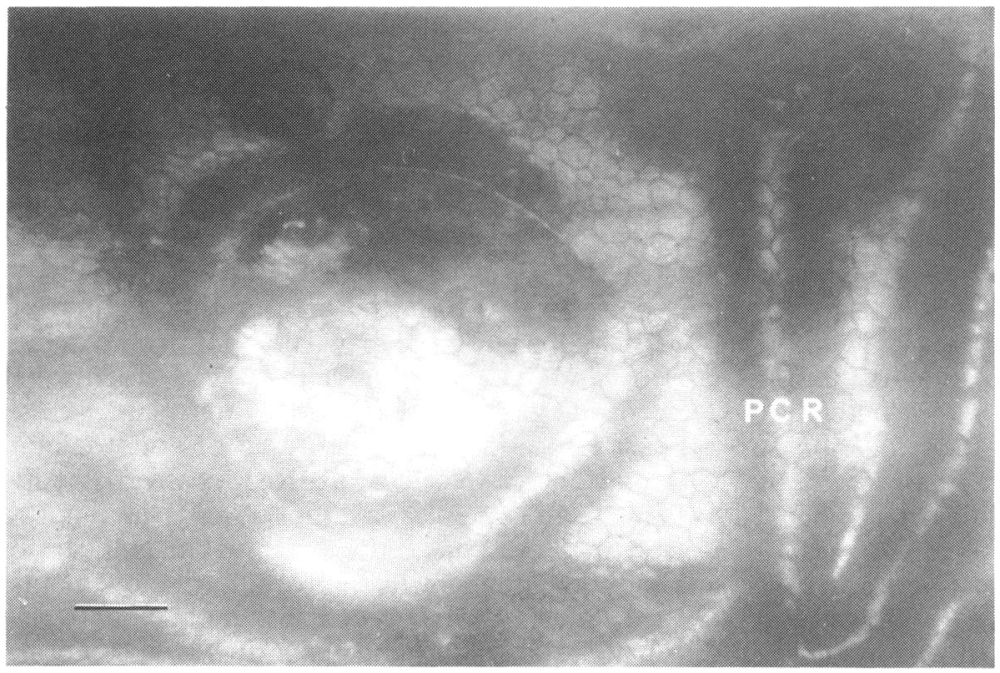

Fig. 6 As in Fig. 5 but on day 21 . The form of the PCR segment included varies slightly in the 2 photographs (Figs. 5 and 6), but clearly identifies the same area (i.e., 5 o'clock on PCRI). Where visible in Figs. 5 and 6 the cells are unchanged in number and size but in general appear more rounded in Fig. 6 than in Fig. $5 . B a r=0.1 \mathrm{~mm}$. 
Fig. 7 Case 2. Specular photomicrograph from the left eye showing small circular lesions in the corneal endothelium. On day 0. Bar $=0.1 \mathrm{~mm}$. Numbers identify same lesion as in Fig. 8.
Fig. 8 As in Fig. 7 but on day 21; area moved slightly to the left and upward. The complex branchings of the PCR clearly identify the same area in Figs. 7 and 8 . Numbers identify the same lesions in both Figs. 7 and $8 . B a r=0.1 \mathrm{~mm}$.
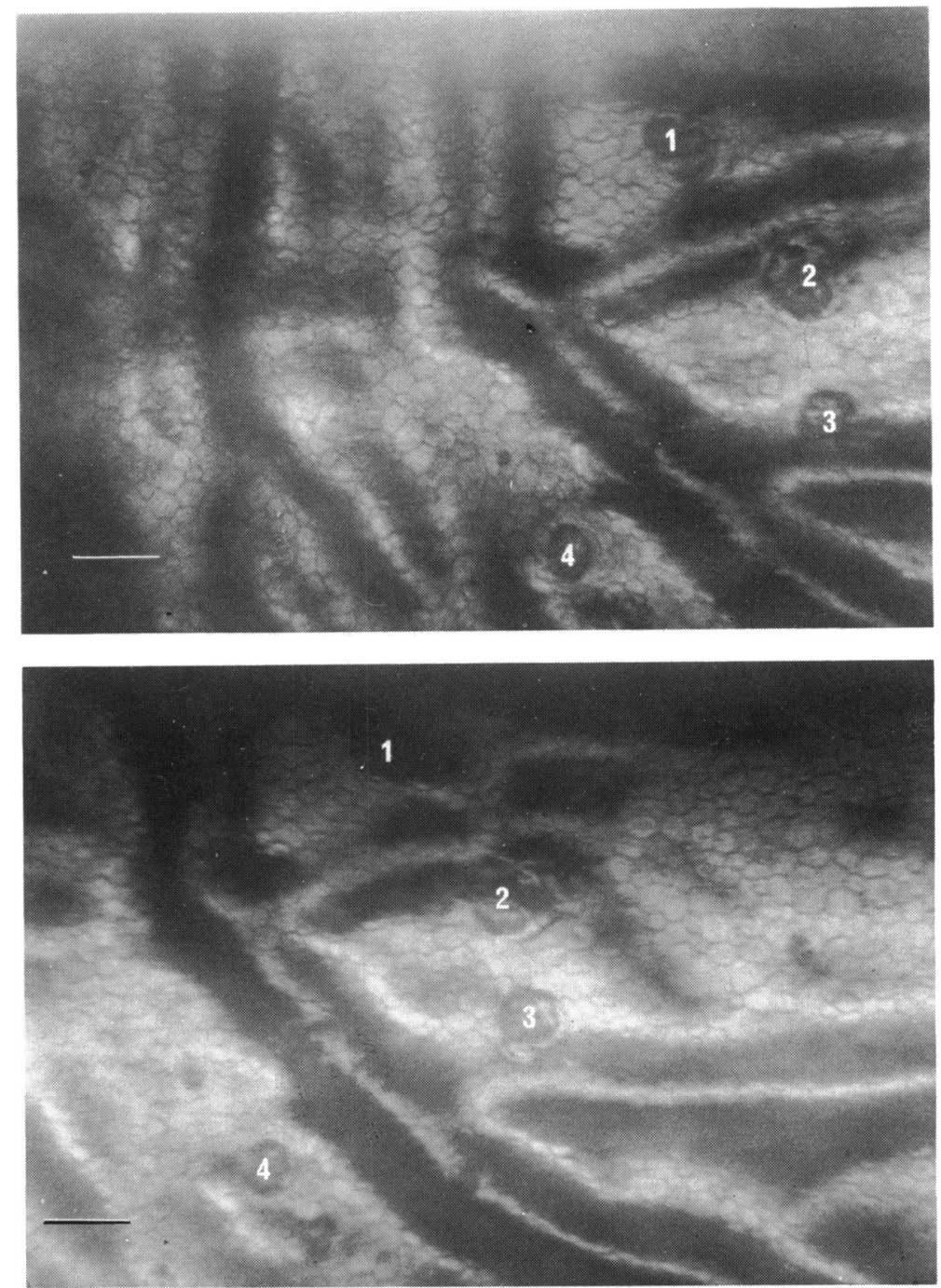

pictures (Figs. 12 and 13), and close comparative examination reveals that, apart from very slight changes in their shapes, the cells are also the same in both photographs.

\section{Discussion}

It is not the purpose of this communication to attempt clinical diagnoses from the illustrations presented but to employ them to demonstrate that the relocation of specific areas of the corneal endothelium of the eye in vivo is possible, indeed simple, with a large-field, contact clinical specular microscope.

Applanation of the cornea induces the formation of the fixed target system of the posterior corneal rings, and the large field readily reveals the relation- ship of an area of the endothelium, with or without a lesion, to the nearest PCR segment, and thus allows easy, rapid, and precise relocation of that area by reference to the PCR system. Although PCRs are induced by the small-field contact microscopes, they may be separated from the area of endothelium of primary interest by more than a field width, and in the presence of the continuous small eye movements the 2 are considerably more difficult to interrelate.

Exact relocation of a given area of endothelium with noncontact specular microscopes is reportedly extremely difficult unless a large, obvious 'landmark' is present. This is due to the low initial magnification and the totally uncontrolled involuntary movements of the eye which prevent recognition of cell patterns or small lesions sufficiently clearly for relocation. 


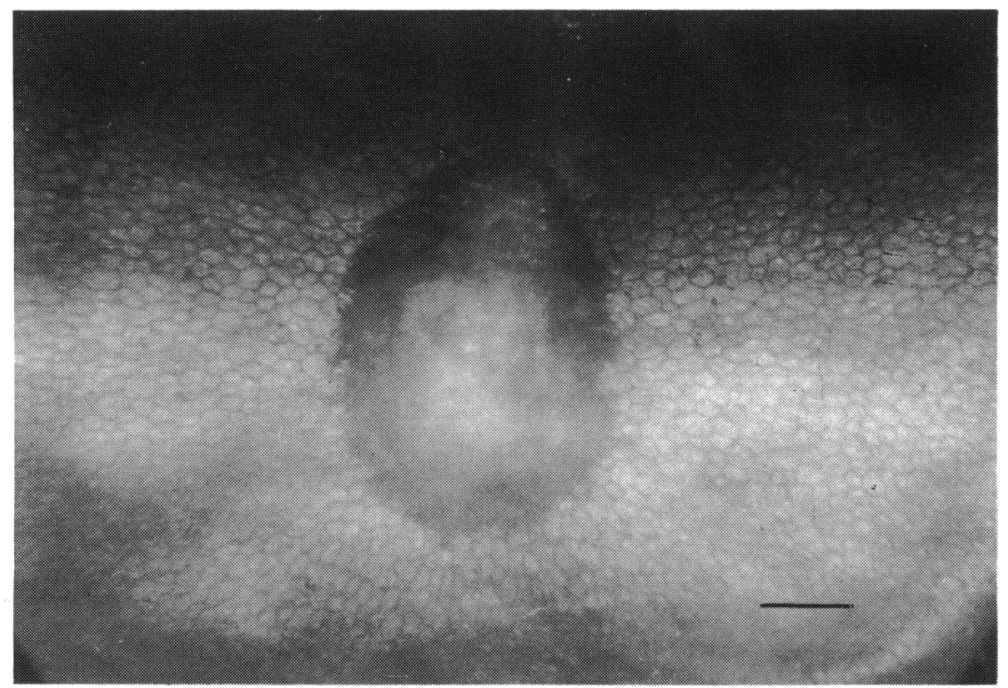

Fig. 9 Case 3. Specular photomicrograph from eye with heterochromic cyclitis, showing very large, apparently acellular lesion; adjacent cells appear stretched towards it. On day 0. $B a r=0 \cdot 1 \mathrm{~mm}$.

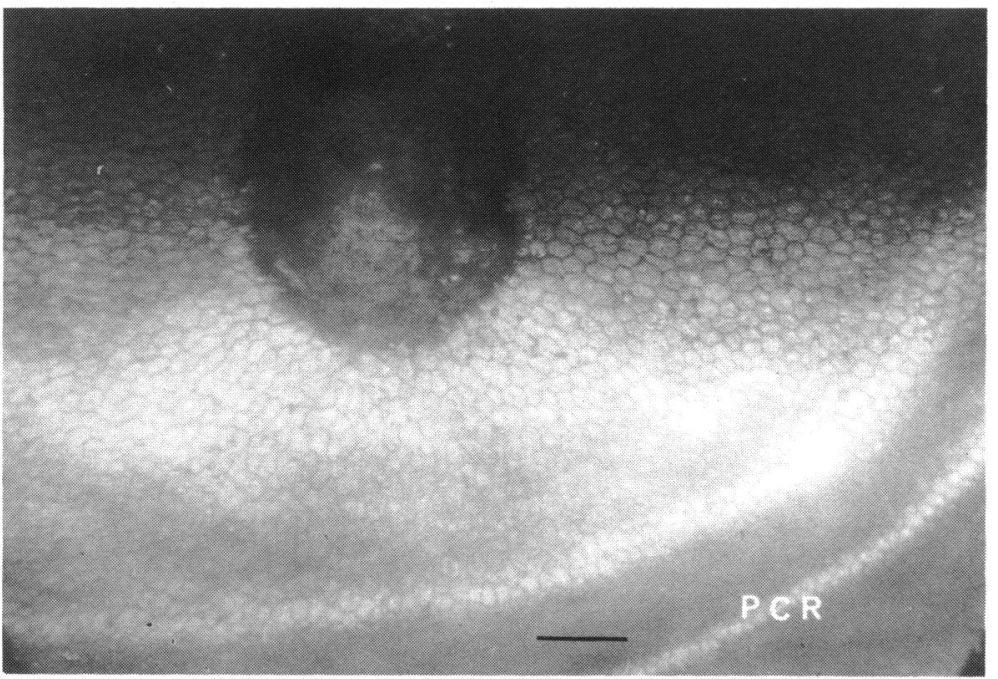

Fig. 10 As in Fig. 9 but off-centred to show position relative to $P C R$. Bar $=0 \cdot 1 \mathrm{~mm}$.

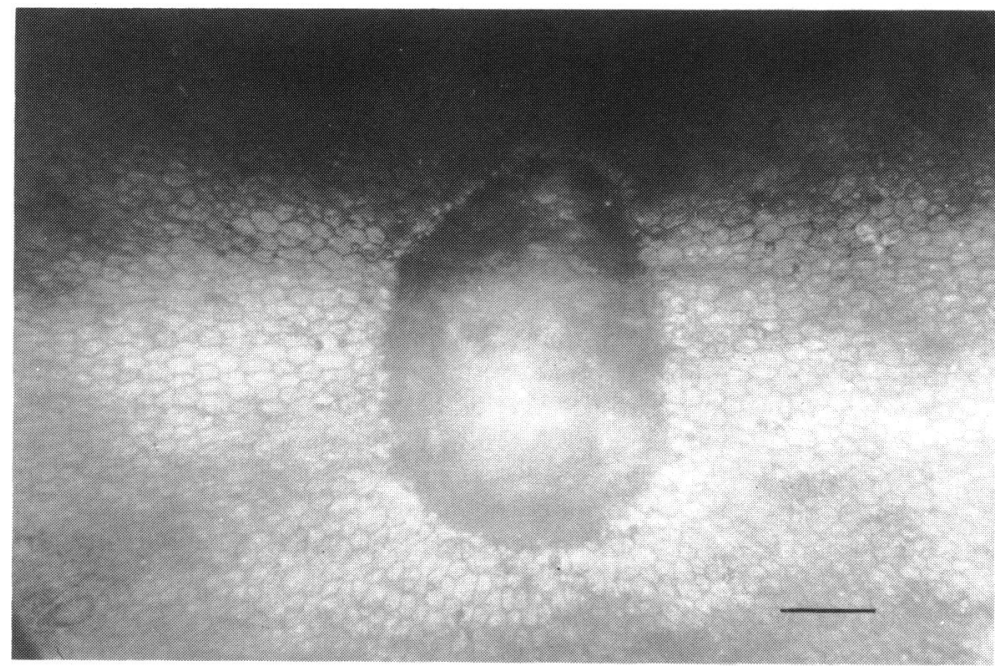

Fig. 11 As in Figs. 9 and 10 but on day 228. The lesion is seen to have enlarged slightly (compare Fig. 9). Bar $=0.1 \mathrm{~mm}$. 
Fig. 12 Case 4. Specular photomicrograph from a normal corneal endothelium. On day 0. Bar $=0.1 \mathrm{~mm}$. The bright vertical streak is a photographic artefact.
Fig. 13 As in Fig. 12 but on day 270. The PCR and other wrinkles are very similar in form in both Figs. 12 and 13, and the cells, although showing small changes in shape, are directly comparable in the 2 pictures. Those marked with black squares serve as a starting point for such comparison.
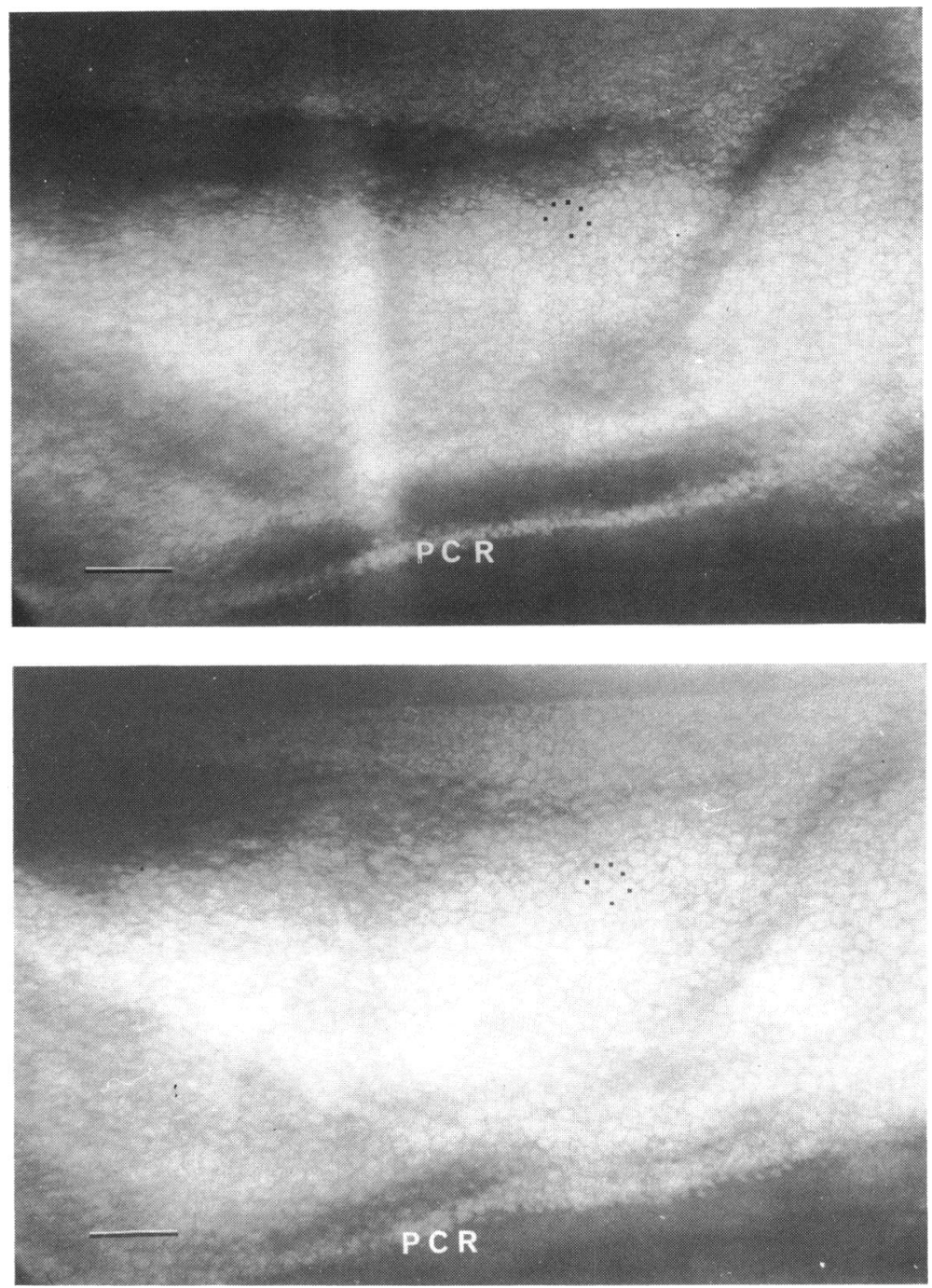

usually necessary to rely on photographic enlargements of the areas photographed to determine whether or not relocation has been achieved.

The cases illustrated here include a range of examples from the relocation of the same area of a normal endothelium to the reidentification of specific large lesions among many of similar appearance. The close approximation of the areas of the endothelium actually included in the paired specular photomicrographs demonstrates the high degree of control of the apparatus that is now possible and that therefore the 'hit or miss' element of the technique has been eliminated. This is the result of the large field of view, the relative stillness of the eye, and the presence of the posterior corneal rings.

It will be noted that, although the configuration of 
the posterior corneal rings, where present, in the pairs of illustrations given is basically the same, there are differences in detail. This is due to slight variations in applanation pressure, intraocular tension, and corneal rigidity. Corneal rigidity is artificially increased by the soft contact lens used during examination; this factor is further dependent upon the fit of the lens, its material, thickness, and degree of hydration. Even so, the basic forms of the PCRs are perfectly adequate for relocation. The degree of reproduction here illustrated is in fact unnecessarily high for this purpose.

These same variations have no visible effect on the morphology of the endothelium as seen when the same area of cells is observed through high-watercontent contact lenses of different materials, such as Duragel 75 and Optima 81, at different degrees of hydration, for example, after prolonged periods of lens exposure or eye closure, and at different pressures of applanation.
We thank Mr Peter Wright for referring cases 2 and 3. William $\mathrm{Ng}$ and Robert Tapper for the photographic processing. and Winifred Pennel for typing the manuscript.

\section{References}

1 Rosenblum P, Stark WJ, Maumenee IH, Hirst LW, Maumenee AE. Hereditary Fuchs' dystrophy. Am J Ophthalmol 1980; 90: 456-62.

2 Sherrard ES, Buckley RJ. Endothelial wrinkling-a complication of clinical specular microscopy. In: The Cornea in Health and Disease. Trans VIth Congress of European Society of Ophthalmology. 1981; London: Academic Press, and Royal Society of Medicine Series 40: 69-74.

3 Sherrard ES, Buckley RJ. Contact clinical specular microscopy of the corneal endothelium: optical modifications to the applanating objective cone. Invest Ophthalmol Visual Sci 1981; 20: 816-20.

4 Sherrard ES, Buckley RJ. Revised optical system for clinical specular microscopy. Proc Int Soc Eve Res Abstracts 1980; 1: 13.

5 Buckley RJ, Sherrard ES. Posterior corneal rings in relation to corneal profile and rigidity. Proc Int Soc Eve Res Abstracts 1980; 1: 14.

6 Olsen T. Non-contact specular microscopy of the human corneal endothelium. Acta Ophthalmol (Kbh) 1979: 57: 986-97. 